\section{$\underset{20}{20}$}

\title{
Prestroke physical activity and early functional status after stroke
}

\author{
N Stroud, ${ }^{1}$ T M L Mazwi, ${ }^{1}$ L D Case, ${ }^{2}$ R D Brown Jr, ${ }^{3}$ T G Brott, ${ }^{1}$ B B Worrall, ${ }^{4}$ \\ J F Meschia, ${ }^{1}$ for the Ischemic Stroke Genetics Study Investigators
}

\begin{abstract}
See Editorial Commentary, p 942

- Additional supplementary tables are published online only at http://jnnp.bmj.com/content/ vol80/issue9

${ }^{1}$ Department of Neurology, Mayo Clinic, Jacksonville, Florida, USA; ${ }^{2}$ The Division of Public Health Sciences, Wake Forest University School of Medicine, Winston-Salem, North Carolina, USA; ${ }^{3}$ The Department of Neurology, Mayo Clinic,

Rochester, Minnesota, USA;

${ }^{4}$ Department of Neurology,

University of Virginia,

Charlottesville, Virginia, USA
\end{abstract}

Correspondence to:

Dr J F Meschia, Department of

Neurology, Mayo Clinic, 4500

San Pablo Road, Jacksonville,

FL 32224, USA; meschia.

james@mayo.edu

Received 9 December 2008

Revised 18 March 2009

Accepted 29 March 2009

\begin{abstract}
Background: The importance of physical activity as a modifiable risk factor for stroke in particular and cardiovascular disease in general is well documented. The effect of exercise on stroke severity and stroke outcomes is less clear. This study aimed to assess that effect. Methods: Data collected for patients enrolled in the Ischemic Stroke Genetics Study were reviewed for prestroke self-reported levels of activity and four measures of stroke outcome assessed at enrolment and approximately 3 months after enrolment. Logistic regression was used to assess the association between physical activity and stroke outcomes, unadjusted and adjusted for patient characteristics.
\end{abstract}

Results: A total of 673 patients were enrolled; $50.5 \%$ reported aerobic physical activity less than once a week, $28.5 \%$ reported aerobic physical activity one to three times weekly, and $21 \%$ reported aerobic physical activity four times a week or more. Patients with moderate and high levels of physical activity were more likely to have higher Barthel Index (BI) scores at enrolment. A similar association was detected for exercise and good outcomes for the Oxford Handicap Scale (OHS). After 3 months of follow-up, moderate activity was still associated with a high BI score. No significant association was detected for activity and the OHS or Glasgow Outcome Scale at followup after adjustment for patient characteristics.

Conclusions: Higher levels of self-reported prestroke physical activity may be associated with functional advantages after stroke. Our findings should be seen as exploratory, requiring confirmation, ideally in a longitudinal study of exercise in an older population.

Stroke is a leading cause of morbidity and mortality, especially among older people. In 2002, cerebrovascular disease ranked second in causes of death worldwide, and that ranking is expected to remain by 2030. ${ }^{1}$ Observational studies have provided strong evidence of a consistent association between physical activity and stroke risk. ${ }^{2-9}$ Additionally, a randomised controlled trial that evaluated the effect of a 3-month home-based physical fitness walking programme found a significant decrease in stroke risk in the walking group on the basis of a 10-year risk estimate using the Framingham risk equation. ${ }^{10}$ The effect of physical activity may partly be mediated through its effect on other risk factors for stroke. ${ }^{11}$

Physical activity has several effects that are potentially beneficial from the perspective of cerebrovascular disease. It has been shown to lower blood pressure and improve lipid profiles. ${ }^{12}$ As expected, physical activity is key in weight loss and its maintenance, ${ }^{12}$ but physical activity has also been shown to increase insulin sensitivity and ultimately decrease the risk of developing diabetes. ${ }^{13-15}$ Physical activity also improves endothelial function, ${ }^{16}$ which enhances vasodilation and vasomotor function in the vessels. In addition, physical activity can play an antithrombotic role by reducing blood viscosity, ${ }^{17}$ fibrinogen levels ${ }^{18}$ and platelet aggregability, ${ }^{19}$ and by enhancing fibrinolysis, ${ }^{20}$ all of which might reduce risk for cerebrovascular events.

The effect, if any, of prestroke physical activity on early poststroke functional status in humans is not well known. The aim of this study was to test the hypothesis that leisure-time physical activity results in favourable effects on functional status and neurological impairment.

\section{METHODS}

We used data from the Ischemic Stroke Genetics Study (ISGS). ISGS is a multicentre inception cohort study of subjects with first-ever ischaemic stroke. The protocol has been described previously. ${ }^{21}$ All participants provided written informed consent to participate in ISGS, and data collection was approved by each site's institutional review board. Participating centres were instructed to screen patients consecutively.

All available medical records pertaining to the stroke evaluation were compiled in standardised fashion, stripped of personal health identifiers and centrally reviewed by a single neurologist for the purpose of assessing stroke subtype diagnoses using multiple standardised criteria. The medical records reviewer completed a stroke work-up checklist, focusing on brain imaging, structural and electrical cardiac evaluation, and cervical and intracranial vascular imaging. Physical activity was assessed using a standardised questionnaire with three categories to determine the patient's activity over the preceding year. Physical activity was defined by actions that produced sweating or a noticeable increase in heart rate analogous to what has been done in the National Health and Nutrition Examination Survey. ${ }^{22}$ Leisure-time physical activity levels were divided into low (vigorous activity sufficient to break a sweat or noticeably raise heart rate less than once a week), moderate (vigorous activity sufficient to break a sweat or noticeably raise heart rate one to three times a week) or high (vigorous activity sufficient to break a sweat or noticeably raise heart rate four or more times a week). Infarct size was determined on the basis of magnetic resonance (MR) imaging scan when available and the computed tomographic scan otherwise. At enrolment (date consent signed) 
and approximately 3 months thereafter, functional status was assessed using the Oxford Handicap Scale (OHS), ${ }^{23}$ Barthel Index (BI) ${ }^{24}$ and Glasgow Outcome Scale (GOS) ${ }^{25}$ Neurological impairment at enrolment was assessed using the National Institutes of Health Stroke Scale (NIHSS). ${ }^{26}$ Baseline assessments were done face to face, and 3-month assessments were done by telephone interview.

Patient and stroke characteristics are summarised using frequencies and percentages, and $\chi^{2}$ tests were used to assess differences in these variables between patients in the three physical activity levels. Stroke severity was quantified by the NIHSS, BI, OHS, GOS and infarct size at baseline and by the BI, $\mathrm{OHS}$ and GOS at follow-up. We dichotomised each measure into "good" and "bad" groups according to the following cutpoints. For BI (possible range of values, 0-100), values of 95 and higher were considered good; for GOS (range 1-5), a value of 1 was considered good; for OHS (range $0-5$ ), values of 0 and 1 were considered good; and for NIHSS (range 0-42), values between 0 and 5 were considered good. The selection of values of 0 to 5 as good outcomes on NIHSS was based on prior studies in which these values predicted discharge disposition after hospitalisation. ${ }^{27}$ These same cut-points were used at follow-up, except for OHS, where a value of 0 was considered good. Infarct size was determined on the basis of maximal cross-sectional diameter of the zone of restricted diffusion as seen on diffusionweighted imaging (or zone of hyperintensity seen on fluidattenuated inversion recovery, if late presentation caused resolution of the area of restricted diffusion) for patients who had $M R$ imaging. For patients who did not have MR imaging, infarct size was determined by the maximal cross-sectional diameter of the zone of hypointensity representing acute infarction. Infarcts smaller than $1.5 \mathrm{~cm}$ were considered small, and those $1.5 \mathrm{~cm}$ and larger were considered large. The dichotomisations were selected to summarise findings in clinically interpretable ways and were selected before statistical analysis. A $\chi^{2}$ test was used to assess the association between physical activity level and the number of bad outcomes (of NIHSS, GOS, OHS and BI). Logistic regression was then used to assess the association between physical activity level and functional status, adjusted for patient and stroke characteristics. A hierarchical approach was used where we included progressively more covariates in subsequent models. Initially, we adjusted for age, body mass index, site, race, sex, primary and secondary smoking exposure; alcohol use; sibling history of stroke; history of diabetes, transient ischaemic attack (TIA), congestive heart failure, myocardial infarction, atrial fibrillation, and hypertension; prior aspirin and anticoagulant use; and glucose levels. Sibling history of stroke was included because it had previously been shown to influence stroke severity in this population. ${ }^{28}$ These variables were included in the model as categorical variables, as shown in supplementary table 1 . The next model also included the prestroke OHS score. We believed that the prestroke OHS score would be highly correlated with the physical activity level, and which came first would not be clear. Stroke characteristics were then added to the previous model. These included the Trial of Org 10172 in Acute Stroke Treatment (TOAST) and Oxfordshire subtypes and thrombolysis within $24 \mathrm{~h}$ after the stroke. A backward-stepping algorithm was then used on the last model to remove variables from the model that were not significant. Results for physical activity are reported as odds ratios (ORs) and 95\% CIs. In all models, the low physical activity group was used as the reference cell. SAS Version 9.1 for Windows was used for all analyses (SAS Institute, Cary, North Carolina).

\section{RESULTS}

A total of 673 patients with first-ever ischaemic stroke were enrolled in ISGS between December 2002 and November 2007. The demographic and clinical characteristics are summarised by physical activity level in supplementary table 1 . Those with the lowest level of physical activity tended to be slightly older; $45 \%$ of those in the low activity group were aged 70 years or older, compared with $31 \%$ of the moderate group and $35 \%$ of the high group $(p=0.002)$. In addition, those with the lowest level of physical activity were more likely to be female $(p<0.001)$ and black $(p=0.003)$ and to have higher levels of heart failure $(p=0.002)$ and hypertension $(p<0.001)$.

As described in the Methods, the OHS, GOS, BI and NIHSS were all dichotomised into good and bad categories according to instrument-specific cut-points. These measures are summarised by activity status in supplementary table 1 . In all cases, the proportion of good responses differed significantly between the activity level groups ( $p<0.05$ in each case). Of the 673 ischaemic patients with stroke, $33 \%$ were good on all four measures, $11 \%$ were good on three, $14 \%$ were good on two, $21 \%$ were good on one, and $21 \%$ were bad on all four measures. Those patients who had performed low activity in the year before the stroke were significantly more likely to have one or more bad outcomes $(p<0.001)$. Overall, $37 \%$ of the patients had small infarcts $(<1.5 \mathrm{~cm})$. There was no significant association between infarct size and physical activity.

The results of logistic regression to assess the association between physical activity and stroke outcomes at baseline in the fully adjusted model are shown in table 1 . Unadjusted results and results adjusted using various stepwise models that included patient characteristics are summarised in supplementary table 2. Low activity was treated as the reference group in each of the comparisons. Unadjusted results showed significant results for all baseline outcome measures. In all models for GOS, OHS and BI, the odds of a good outcome were higher for patients who reported moderate to high prestroke physical activity levels compared with those who reported low physical activity. There was little difference in outcomes between those with moderate and those with high activity $(\mathrm{p}>0.05)$. Adjusted for patient characteristics, the association of physical activity with GOS and NIHSS became non-significant. In addition, the association between physical activity and infarct size remained non-significant. However, the association between physical activity and the baseline poststroke OHS and the association between physical activity and the baseline poststroke BI remained significant or borderline significant for all adjusted models. There was no significant association between physical activity and infarct size.

The results of logistic regression to assess the association between physical activity and stroke outcomes at 3 months after stroke in the fully adjusted model are shown in table 1 . Unadjusted results and results adjusted using various stepwise models that included patient characteristics are summarised in supplementary table 3. Unadjusted, moderate and high levels of physical activity were significantly associated with better GOS, OHS and BI scores. After adjustment for patient and stroke characteristics, only the association between physical activity and BI remained significant.

\section{DISCUSSION}

Our results showed an association between physical activity and higher functional status after first-ever stroke for two of four measures of stroke outcome at baseline. Better results for functional status after stroke could not be explained by differences in age, body mass index, race or sex, substance use or family history; premorbid functional status and medical 
Table 1 Association between leisure-time physical activity and good stroke outcomes at enrolment (baseline) and 3 months after the stroke

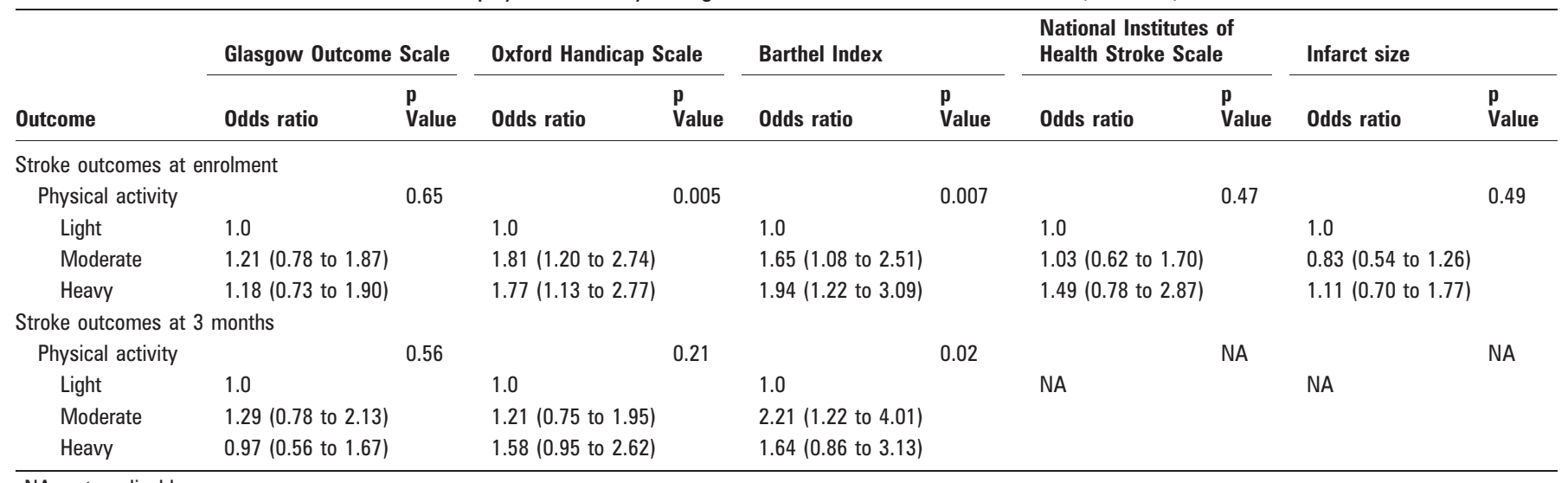

NA, not applicable.

conditions (diabetes and glucose tolerance, myocardial infarction, hypertension, congestive heart failure, TIAs and atrial fibrillation); medications (aspirin and anticoagulants); or stroke subtype or use of thrombolysis. High levels of physical activity nearly doubled the odds that patients would have a favourable $\mathrm{BI}$ or OHS score at the time of study enrolment. For the BI, the effect was slightly less for moderate compared with high activity levels; this was not the case for the OHS. Deplanque and colleagues ${ }^{29}$ also found a trend toward an exercise durationdependent significant association of physical activity and outcome for exercise duration of more than $2 \mathrm{~h}$ weekly when using the NIHSS, a modified Rankin scale and the BI.

Unadjusted data from 3 months after the stroke were significant for better functional status scores for GOS, OHS and BI scales with increasing exercise levels. On adjustment, however, only the association for the BI for moderate exercise remained significant (supplementary table 3). We believe that the trends identified are accurate but that greater study power may be necessary to better delineate the positive associations of functional status with prestroke exercise habits.

This study has several limitations. The first is the use of selfreported physical activity data, which may be of doubtful accuracy. It is possible that subjects who do well report higher levels of prestroke activity (recall bias). However, several studies have shown that self-reporting is a reproducible and reliable way to assess patients' activity levels. ${ }^{30-32}$ The second limitation relates to the ability to generalise from these results. The study was a retrospective analysis, all patients were evaluated at dedicated stroke centres, and all included cases representing first-ever ischaemic stroke. Because this study used data obtained from patients enrolled in an ischaemic stroke genetics study that required written informed consent, the study population tends to skew mild in severity of deficit. This problem is compounded by the fact that one of the five ISGS centres did not allow enrolment of subjects by surrogate consent. This led to consent bias toward subjects with milder strokes. ${ }^{33}$ Due to the eligibility criteria of ISGS, our findings do not apply to patients with recurrent strokes or haemorrhagic strokes. A third limitation is that prestroke OHS is an imperfect measure of prestroke health status. Other covariates such as depression, socio-economic status and comorbid conditions like arthritis undoubtedly contribute to prestroke health status but were not captured or were not captured in all participants. Finally, four outcome measures were assessed in this study, making it possible for false-positive findings to emerge from multiple testing.

Our findings should be regarded as exploratory because of the potential for recall bias and limitations on generalizability. A longitudinal study of exercise that followed initially stroke-free individuals before, during and after stroke would be ideal for addressing the methodological limitations of our study.

There are several potential reasons why exercise might improve functional outcomes. Active individuals have greater cardiovascular reserve and may demonstrate better haemodynamics after a stroke, better collateralisation of flow after arterial occlusions and better oxygen extraction from the blood as a result of changes to the oxygen dissociation curve. Cardiovascular reserve may minimise loss of the ischaemic penumbra in the acute phase of stroke, resulting in better functional status. Individuals with higher activity levels may also have greater functional neuromuscular reserves and as a result may be better able to compensate for deficits, thus minimising the effects of disabling strokes. Studies have shown a positive relationship between physical activity and the enhanced release of nitric oxide and expression of endothelial nitric oxide synthase (eNOS). ${ }^{34-36} \mathrm{~A}$ study using wild-type mice demonstrated the neuroprotective effect of this very relationship. Mice with the eNOS gene had a smaller cerebral infarct size, milder neurological deficits and improved regional blood flow and vasodilation after a 3-week exercise programme compared with controls. ${ }^{37}$ This effect is thought to be due to regulation of blood pressure, local blood flow, vasodilation, and platelet aggregation and adhesion.

\section{CONCLUSIONS}

These data show that aerobic physical activity may improve functional status immediately after stroke. Our findings warrant independent confirmation in light of the limitations of study design. We infer that physical activity may have implications in stroke that go beyond the role it has in reversing risk factors. Patients who are active may recover more quickly immediately after a stroke, with trends that point to better outcomes at the 3-month follow-up. The amount of activity that may be required to show a positive effect is unknown.

Funding: The Ischemic Stroke Genetics Study (ISGS) was supported by a grant from the NINDS (NINDS R01 NS42733; JFM, principal investigator).

\section{Competing interests: None.}

Ethics approval: Ethics approval was provided by the Institutional Review Boards governing Mayo Clinic (Rochester, Minnesota and Jackson, Florida), Emory University School of Medicine (Atlanta, Georgia), the University of Florida/Shands Hospital (Jacksonville, Florida), the University of Virginia (Charlottesville, Virginia) and Wake Forest University School of Medicine (Winston-Salem, North Carolina).

Patient consent: Obtained.

Statistical analysis was conducted by LDC, Department of Biostatistical Sciences, Division of Public Health Sciences, Wake Forest University School of Medicine. 


\section{REFERENCES}

1. Mathers CD, Loncar D. Projections of global mortality and burden of disease from 2002 to 2030. PLoS Med 2006;3:e442.

2. Abbott RD, Rodriguez BL, Burchfiel CM, et al. Physical activity in older middle-aged men and reduced risk of stroke: the Honolulu Heart Program. Am J Epidemiol 1994;139:881-93.

3. Gillum RF, Mussolino ME, Ingram DD. Physical activity and stroke incidence in women and men: the NHANES I Epidemiologic Follow-up Study. Am J Epidemiol 1996;143:860-9

4. Lee IM, Paffenbarger RS Jr. Physical activity and stroke incidence: the Harvard Alumni Health Study. Stroke 1998;29:2049-54.

5. Sacco RL, Gan R, Boden-Albala B, et al. Leisure-time physical activity and ischemic stroke risk: the Northern Manhattan Stroke Study. Stroke 1998;29:380-7.

6. Hu G, Sarti C, Jousilahti $P$, et al. Leisure time, occupational, and commuting physical activity and the risk of stroke. Stroke 2005:36:1994-9.

7. Lindenstrom E, Boysen G, Nyboe J. Lifestyle factors and risk of cerebrovascular disease in women: the Copenhagen City Heart Study. Stroke 1993:24:1468-72.

8. Krarup LH, Truelsen T, Pedersen A, et al. Level of physical activity in the week preceding an ischemic stroke. Cerebrovasc Dis 2007;24:296-300.

9. Lee IM, Sesso HD, Oguma $Y$, et al. The "weekend warrior" and risk of mortality. Am J Epidemiol 2004;160:636-41.

10. Tully MA, Cupples ME, Chan WS, et al. Brisk walking, fitness, and cardiovascular risk: a randomized controlled trial in primary care. Prev Med 2005;41:622-8.

11. Gorelick PB, Sacco RL, Smith DB, et al. Prevention of a first stroke: a review of guidelines and a multidisciplinary consensus statement from the National Stroke Association. JAMA 1999;281:1112-20

12. Wood PD, Stefanick ML, Dreon DM, et al. Changes in plasma lipids and lipoproteins in overweight men during weight loss through dieting as compared with exercise. N Engl J Med 1988;319:1173-9.

13. Hu FB, Sigal RJ, Rich-Edwards JW, et al. Walking compared with vigorous physical activity and risk of type 2 diabetes in women: a prospective study. JAMA 1999:282:1433-9.

14. Devlin JT. Effects of exercise on insulin sensitivity in humans. Diabetes Care 1992:15:1690-3.

15. Henriksson J. Influence of exercise on insulin sensitivity. J Cardiovasc Risk 1995;2:303-9.

16. Laufs U, Wassmann $\mathrm{S}$, Czech $\mathrm{T}$, et al. Physical inactivity increases oxidative stress, endothelial dysfunction, and atherosclerosis. Arterioscler Thromb Vasc Biol 2005:25:809-14.

17. Koenig W, Sund M, Doring A, et al. Leisure-time physical activity but not workrelated physical activity is associated with decreased plasma viscosity: results from a large population sample. Circulation 1997:95:335-41.

18. Ernst E. Regular exercise reduces fibrinogen levels: a review of longitudinal studies. Br J Sports Med 1993:27:175-6.
19. Rauramaa R, Salonen JT, Seppanen K, et al. Inhibition of platelet aggregability by moderate-intensity physical exercise: a randomized clinical trial in overweight men Circulation 1986;74:939-44.

20. Boman K, Hellsten G, Bruce A, et al. Endurance physical activity, diet and fibrinolysis. Atherosclerosis 1994;106:65-74.

21. Meschia JF, Brott TG, Brown RD Jr, et al. The Ischemic Stroke Genetics Study (ISGS) Protocol. BMC Neurol 2003:3:4.

22. Anon. Questionnaire: physical activity and physical fitness-PAO. Atlanta: Department of Health and Human Services, Centers for Disease Control and Prevention. http://www. cdc.gov/nchs/data/nhanes/nhanes 05 06/SP paq d.pdf (accessed 19 Aug 2008).

23. Bamford JM, Sandercock PA, Warlow CP, et al. Interobserver agreement for the assessment of handicap in stroke patients. Stroke 1989;20:828.

24. Mahoney FI, Barthel DW. Functional evaluation: the Barthel Index. Md State Med J 1965; 14:61-5.

25. Kasner SE. Clinical interpretation and use of stroke scales. Lancet Neurol 2006;5:603-12.

26. Brott T, Adams HP Jr, Olinger CP, et al. Measurements of acute cerebral infarction: a clinical examination scale. Stroke 1989:20:864-70.

27. Schlegel DJ, Tanne D, Demchuk AM, et al. Prediction of hospital disposition afte thrombolysis for acute ischemic stroke using the National Institutes of Health Stroke Scale. Arch Neurol 2004;61:1061-4.

28. Meschia JF, Case LD, Worrall BB, et al. Family history of stroke and severity of neurologic deficit after stroke. Neurology 2006:67:1396-402.

29. Deplanque D, Masse I, Lefebvre C, et al. Prior TIA, lipid-lowering drug use, and physical activity decrease ischemic stroke severity. Neurology 2006;67:1403-10.

30. Kurtze N, Rangul V, Hustvedt BE, et al. Reliability and validity of self-reported physical activity in the Nord-Trondelag Health Study: HUNT 1. Scand J Public Health 2008;36:52-61.

31. Teixeira-Salmela LF, Devaraj R, Olney SJ. Validation of the human activity profile in stroke: a comparison of observed, proxy and self-reported scores. Disabil Rehabil 2007:29:1518-24.

32. Kurtze N, Rangul V, Hustvedt BE, et al. Reliability and validity of self-reported physical activity in the Nord-Trondelag Health Study (HUNT 2). Eur J Epidemiol 2007:22:379-87.

33. Chen DT, Case LD, Brott TG, et al. Impact of restricting enrollment in stroke genetics research to adults able to provide informed consent. Stroke 2008;39:831-7.

34. Hornig B, Maier V Drexler H. Physical training improves endothelial function in patients with chronic heart failure. Circulation 1996;93:210-14.

35. Sessa WC, Pritchard K, Seyedi N, et al. Chronic exercise in dogs increases coronary vascular nitric oxide production and endothelial cell nitric oxide synthase gene expression. Circ Res 1994;74:349-53

36. Fukai T, Siegfried MR, Ushio-Fukai M, et al. Regulation of the vascular extracellular superoxide dismutase by nitric oxide and exercise training. J Clin Invest 2000; 105:1631-9.

37. Endres M, Gertz K, Lindauer U, et al. Mechanisms of stroke protection by physical activity. Ann Neurol 2003;54:582-90.

\section{Access the latest content chosen by our Editors}

BMJ Journals editors select an article from each issue to be made free online immediately on publication. Other material is free after 12 months to non-subscribers. Access the Editor's Choice from the home page - or expand your horizons and see what the other BMJ Journals editors have chosen by following the links on any BMJ Journal home page. 